\title{
Self-reported adherence to physical activity recommendations compared to the IPAQ interview in patients with hypertension
}

This article was published in the following Dove Medical Press journal: Patient Preference and Adherence

\author{
Glaube R Riegel' \\ Giulia B Martins' \\ Afonso G Schmidt' \\ Marcela P Rodrigues' \\ Gerson S Nunes ${ }^{2}$ \\ Vicente Correa Jr ${ }^{1,2}$ \\ Sandra C Fuchs' \\ Flavio D Fuchs ${ }^{1,2}$ \\ Paula AB Ribeiro1,3 \\ Leila B Moreira ${ }^{1,4}$ \\ 'Postgraduate Studies Program in \\ Cardiology, School of Medicine, \\ Universidade Federal do Rio \\ Grande do Sul, Porto Alegre, \\ RS, Brazil; ${ }^{2}$ Cardiology Division, \\ Hospital de Clínicas de Porto \\ Alegre, Porto Alegre, RS, Brazil; \\ ${ }^{3}$ Cardiology Division, University of \\ Montreal Hospital Research Centre \\ (CRCHUM), Montréal, QC, Canada; \\ ${ }^{4}$ Pharmacology Department, Instituto \\ de Ciências Básicas da Saúde (ICBS), \\ Universidade Federal do Rio Grande \\ do Sul, Porto Alegre, RS, Brazil
}

Correspondence: Paula AB Ribeiro University of Montreal Hospital Research Centre (CRCHUM), 900 St Denis Street, Montreal, QCH2X 0A9, Canada Tel + I 5I48908000 ext I7I66

Email paula.ribeiro.chum@ssss.gouv.qc.ca
Background: Physical activity (PA) is recommended as adjuvant therapy to control blood pressure (BP). The effectiveness of simple recommendations is not clear. We aimed to assess the agreement between self-report of adherence to PA in clinical routine and International Physical Activity Questionnaire (IPAQ) interview and its association with BP control.

Methods: A cross-sectional study was conducted with hypertensive outpatients. Adherence to recommendation to PA was assessed by the physician and IPAQ interview. A cutoff of 150 minutes/week was used to classify active or nonactive patients. High sitting time was considered $>4$ hours/day. A total of 127 individuals (SBP $144.9 \pm 24.4 \mathrm{mmHg} / \mathrm{DBP}$ $82.0 \pm 12.8 \mathrm{mmHg}$ ) were included.

Results: A total of 69 subjects $(54.3 \%)$ reported to be active to their physician, whereas 81 $(63.8 \%)$ were classified as active by IPAQ $(6.3 \%$ active in leisure time PA). Kappa test was 0.22 (95\% CI, 0.06-0.37). The rate of BP control was $45.7 \%$. There was no association with the reported PA assessed by both methods nor with sitting time. Our results demonstrated poor agreement between self-report adherence and IPAQ interview, and neither evaluation was associated with BP control.

Conclusion: Our findings underpin evidences that a simple PA recommendation has low association with BP control in clinical settings.

Keywords: blood pressure, exercise, treatment adherence, self-report, hypertension, physical activity counseling

\section{Background}

According to WHO statistics, ${ }^{1}$ the estimated prevalence of hypertension worldwide is about 1.13 billion. Meta-analysis ${ }^{2}$ of epidemiological studies estimated the prevalence of $30 \%$ in Brazil. Hypertension has been defined as blood pressure (BP) level equal to or greater than $140 / 90 \mathrm{mmHg},{ }^{3}$ but the recently released AHA-ACC guideline for the prevention, detection, evaluation, and management of $\mathrm{BP}$ in adults ${ }^{4}$ now defines high $\mathrm{BP}$ as $\mathrm{SBP} \geq 130 \mathrm{mmHg}$ or $\mathrm{DBP} \geq 80 \mathrm{mmHg}$. The reduction proposed by the AHA-ACC is in consonance with new evidences about the benefit of reducing BP below that level; ${ }^{4}$ however, it was not incorporated by other guidelines. BP reduction is the primary mechanism to promote the prevention of cardiovascular diseases. Weight control, reduction in salt intake, and improving physical activity (PA) are among the recommended nonpharmacological interventions to control hypertension. ${ }^{5}$

The recommendations for PA in hypertensive patients are relatively consistent. The American College of Sports Medicine guidelines ${ }^{6}$ recommend combined PA interventions to improve health and reduce premature death, primarily endurance PA 
combined with resistance exercise. The European Society of Cardiology ${ }^{7}$ proposes a daily 30 minutes of PA of moderate intensity to treat hypertension. The BP-lowering effect of exercise is recognized by most experts. ${ }^{8}$ A meta-analysis ${ }^{9}$ of clinical trials showed that continuous aerobic exercise promoted a reduction in mean SBP of $-3.84 \mathrm{mmHg}$ and DBPs of $-2.58 \mathrm{mmHg}$. Overall results from meta-analysis (summarized effects) often see positive effect. ${ }^{9-12}$

However, not all evidence converge to demonstrate the BP-lowering efficacy of exercise training and PA. ${ }^{13}$ Is expected to see different effects for exercise and PA counseling, especially when interventions cannot be directly supervised, such as in ACTID study, where PA documented by pedometer was not associated with a reduction in BP. ${ }^{14}$ The translation of the efficacy of exercise programs demonstrated in experimental conditions to the clinical scenario is another key point. In a meta-analysis ${ }^{15}$ of 14 randomized studies, the advice to exercise decreased SBP and DBP in the 6-month follow-up but not at 12-month follow-up. We have repeatedly demonstrated that reported adherence to our team of physicians to engage in PA is not associated with BP-lowering effect. ${ }^{16,17}$ The controversy could be explained by patient's inaccurate understanding on doctor advice or by the subjectivity of self-reported adherence, so whether is a matter of measurement or real low adherence is to be determined. Therefore, different results suggest that the overlap of measurement method and type of intervention could play an important role besides patients' behavior.

Wherefore, the primary objective of this study is to explore the agreement between reported PA adherence to the assistant physician and the interview with long-version International Physical Activity Questionnaire (IPAQ) in a clinical ambulatory routine. The secondary objective was to evaluate the association of PA levels with BP control by both methods.

\section{Methods}

This cross-sectional study was conducted in a convenience sample of individuals aged 18 years or more, who were previously followed for at least 1 year at our reference hypertension clinic and participated in a previous cohort study. ${ }^{17}$ All patients' cohort were eligible for the study, if they have had a scheduled visit from April to September 2015, excluding those unable to or with severe limitations to walk. Data were collected from April to September 2015. The study was approved by the Ethics Committee of Hospital de Clínicas de Porto Alegre, which is accredited by the US Office of Human Research Protections as an Institutional Review Board. All procedures were performed in accordance with the principles of the Declaration of Helsinki, and all participants gave written consent to participate.

\section{Outcome definition}

Hypertension was defined as BP $\geq 140 / 90 \mathrm{mmHg}$ or the use of BP-lowering drugs. Two standardized measures of BP were taken with automatic sphygmomanometer (OMROMCP705, Dupont, France) during consultation, and their average was used in analysis. Patients were sitting with feet resting on the floor and right arm resting at the height of the precordium; normal cuff (up to $32 \mathrm{~cm}$ ) or large cuff $(>33 \mathrm{~cm})$ were used. Minimum of 5 minutes of rest and a 1-minute interval between measurements was given. The patients were recommended to not engage on caffeinated drinks, smoking, or PA at least 30 minutes before consultations, as recommended. ${ }^{4}$

\section{PA measurements}

1) Adherence to recommendation: patients were systematically advised by their physician to walk or perform aerobic exercise for at least 30 minutes, 3-5 days a week, during routine consultations. Adherence to the PA counseling was checked at each consultation by asking the patient "have you following the recommended PA since the last visit?" The self-reported adherence was recorded at each consultation and the most recent was collected for analysis. PA clinical adherence was categorized as yes or no.

2) IPAQ interview: trained investigators applied the $\mathrm{IPAQ}^{18}$ long version immediately before the consultation (all investigators were blinded for the BP measurements). This questionnaire was chosen for being the most used, validated, and specially for having special questions about walking PA at each domain of PA (domestic, transportation, work, and leisure time), which we believed is the most performed type of PA among our patients. ${ }^{18}$ Patients then were classified as active when performed at least 150 minutes of moderate to vigorous PA in the last week, considering the four domains: PA at work, transport-related, housework, and leisure time. Also, sitting time was evaluated as a complementary domain to PA and was defined by the time the individual remained seated (hours/day). Daily-life examples were provided to facilitate self-report, such as sitting at work or home, watching television, or driving a car. ${ }^{19}$

\section{Statistical analyses}

To test the agreement between self-report adherence and IPAQ interview, a sample size of 100 participants was estimated considering the prevalence of $50 \%{ }^{17}$ - adherence to recommended PA, expected kappa 0.70 , K-value of nullity of 
$0.40,90 \%$ power, and a significance level of $5 \%$. Variables were described by mean and SD or absolute and relative frequencies. Student's $t$-test or chi-squared test was applied to compare the groups according to self-report of adherence to the PA recommendation. Kappa statistic was conducted in the PEPI software to analyze the agreement between the methods of PA evaluation, considering the overall score and separated domains for IPAQ. Other analyses were performed on SPSS version 18 (IBM Corporation, Armonk, NY, USA).

\section{Results}

The 127 patients included were $67 \pm 9$ years old, 64\% women, with mean SBP 144.9 $\pm 24.4 \mathrm{mmHg}$ and DBP $82.0 \pm 12.8 \mathrm{mmHg}$. Among all, 69 (54.3\%) subjects reported they were following the recommendation for PA to their physicians, whereas $81(63.8 \%)$ performed moderate to vigorous PA for 150 minutes or more in the last week, according to the IPAQ. The characteristics of total sample according to the self-reported adherence are listed in Table 1. There were no group differences.

The agreement of self-report adherence information with the IPAQ interview was weak (kappa $=0.22 ; 95 \% \mathrm{CI}$, 0.06-0.37). The agreement of IPAQ domains with self-report is presented in Table 2. A variable considering a combination of transport-related and leisure time PA was created, and the correlation was slightly higher (kappa $=0.35 ; 95 \% \mathrm{CI}$, 0.19-0.50).

Considering the total sample $(n=127)$, only $44(34.6 \%)$ participants classified as active by IPAQ reported following medical recommendations and $32(25.2 \%)$ classified as inactive reported not following the recommendation (Table 2). The sitting time ranged from 1.05 to 12.6 hours, with average of $5.4 \pm 2.6$ hours and median of 5.4 hours/day. Of the individuals who remained seated at least 4 hours/day $(n=80)$, $43.8 \%$ (35) reported adherence to medical advice $(P=0.58)$.
The prevalence of controlled BP in the sample was $45.7 \%$ $(\mathrm{n}=58)$. Among participants classified as physically active by IPAQ $(n=55), 46.6 \%$ had controlled BP $(n=27)$ vs $40.6 \%$ $(n=28)$ in the group not active $(n=72)(P=0.37)$. Self-report adherence was $41.4 \%$ vs $46.4 \% ; P=0.57$, respectively. Overall, there was no association of controlled BP or mean SBP and DBP with the self-reported adherence to PA, as well as any association for sitting time. The integrated view about the rate of BP control by method of evaluation and PA status is presented in Figure 1.

\section{Discussion}

In this study, conducted at a specialized hypertension clinic, self-reported adherence to the medical recommendation to perform PA 3-5 days a week showed weak agreement with adherence estimated by the IPAQ interview. There was no association between PA evaluated by both methods and control of SBP or DBP.

The IPAQ has been recommended as an appropriate large-scale method to assess PA in different life domains. Different methods were used to evaluate the IPAQ performance, including self-reporting and objective methods. ${ }^{20,21}$ Considering that in our study the clinical counseling emphasized walking, a greater PA would be expected in domains like transport-related or leisure time. Although those domains taken together have presented stronger agreement with the self-report, the recorded level still reflects low PA.

Our study did not find any association of PA with BP control. Although most clinical trials $\mathrm{s}^{9,11,12,22}$ have identified the efficacy of supervised and structured exercise programs to lower BP, other studies also lack this association ${ }^{13}$ or with an effect restricted to a short period of time. ${ }^{15}$

Different from clinical trials, our observational studies have previously demonstrated ${ }^{16,17}$ the lack of association of a dichotomy self-report of PA adherence and BP outcomes

Table I Clinical characteristics according to self-reported adherence to the medical recommendation to engage in PAs

\begin{tabular}{|c|c|c|c|c|}
\hline Characteristics & $\begin{array}{l}\text { Overall sample } \\
(n=\mid 27)\end{array}$ & $\begin{array}{l}\text { PA adherence } \\
(n=69)\end{array}$ & $\begin{array}{l}\text { No PA adherence } \\
(n=58)\end{array}$ & $P$-value \\
\hline Women & 81 (63.8\%) & $42(60.3 \%)$ & 39 (66.7\%) & 0.29 \\
\hline Age (years) & $67.6 \pm 9.5$ & $67.3 \pm 8.5$ & $67.7 \pm 10.3$ & 0.98 \\
\hline Education (years) & $7.0 \pm 3.1$ & $6.9 \pm 2.9$ & $7.2 \pm 3.3$ & 0.57 \\
\hline Antihypertensives (n) & $3.5 \pm 1.3$ & $3.69 \pm 1.3$ & $3.4 \pm 1.3$ & 0.84 \\
\hline BMI $\left(\mathrm{kg} / \mathrm{m}^{2}\right)$ & $30.2 \pm 5.3$ & $29.2 \pm 5.2$ & $31.0 \pm 5.3$ & 0.75 \\
\hline BP controlled & $56(44.1 \%)$ & $29(4 \mathrm{I} .4 \%)$ & 27 (46.4\%) & 0.35 \\
\hline $\mathrm{SBP}(\mathrm{mmHg})$ & $144.9 \pm 24.43$ & $145.9 \pm 24.29$ & $142.5 \pm 24.9$ & 0.71 \\
\hline $\mathrm{DBP}(\mathrm{mmHg})$ & $82 \pm 12.8$ & $82.3 \pm 13.12$ & $81.1 \pm 12.10$ & 0.59 \\
\hline Follow-up (years) & $12 \pm 5$ & $12.7 \pm 5.2$ & $11.7 \pm 4.8$ & 0.25 \\
\hline IPAQ (minutes/week) ${ }^{\mathrm{a}}$ & $120(45-294)$ & $195(87-34 \mid)$ & $70(30-247)$ & $<0.001$ \\
\hline
\end{tabular}

Notes: Mean \pm SD. a Median (p25-p75).

Abbreviations: PA, physical activity; BMI, body mass index; BP, blood pressure; IPAQ, International Physical Activity Questionnaire. 
Table 2 Agreement between self-reported physical activity adherence and IPAQ interview

\begin{tabular}{l|l|l|l}
\hline IPAQ domains & Kappa $(\mathbf{9 5 \%} \mathbf{C l})$ & $\begin{array}{l}<\mathbf{I 5 0} \text { minutes/week + no } \\
\text { adherence } \\
\mathbf{N}(\%)\end{array}$ & $\begin{array}{l}>\mathbf{I 5 0} \text { minutes/week + } \\
\text { adherence } \\
\mathbf{N} \text { (\%) }\end{array}$ \\
\hline Work & $-0.00(-0.10$ to 0.09$)$ & $64(50.4)$ & $4(3.1)$ \\
TR & $0.20(0.06$ to 0.35$)$ & $61(48)$ & $18(14.2)$ \\
House & $0.1 I(-0.06$ to 0.28$)$ & $42(33.1)$ & $29(22.8)$ \\
Leisure time & $0.15(0.05$ to 0.24$)$ & $69(54.3)$ & $8(6.3)$ \\
TR + LT & $0.35(0.19$ to 0.50$)$ & $60(47.2)$ & $27(21.3)$ \\
Overall & $0.22(0.06$ to 0.37$)$ & $32(25.2)$ & $44(34.6)$ \\
Sitting time $(>4$ hours $)$ & $0.18(0.03$ to 0.32$)$ & $35(27.6)$ & $36(28.4)$ \\
\hline
\end{tabular}

Abbreviations: IPAQ, International Physical Activity Questionnaire; TR, transport-related; LT, leisure time.

in the clinical setting. The current study intents to answer

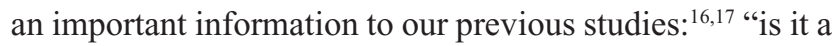
clear lack of association or method limitation?" Our results demonstrate that even a more detailed PA report does not better associate with BP profile in our patients; in sum, a single PA counseling seems not to be effective in improving PA behavior. Physicians' lack of enthusiasm or lack of clarity on the type and duration of exercise could be another limiting factor. Our ambulatory professionals' give PA counseling as routine at every consultation; however, how enthusiastic it can be was not explored in this study. We acknowledge that there is some evidence suggesting that the received PA counseling and what patients really need to perform can be differently perceived among patients, as shown before in other chronic metabolic diseases, ${ }^{23,24}$ which might partially explain our lack of association between PA and BP.

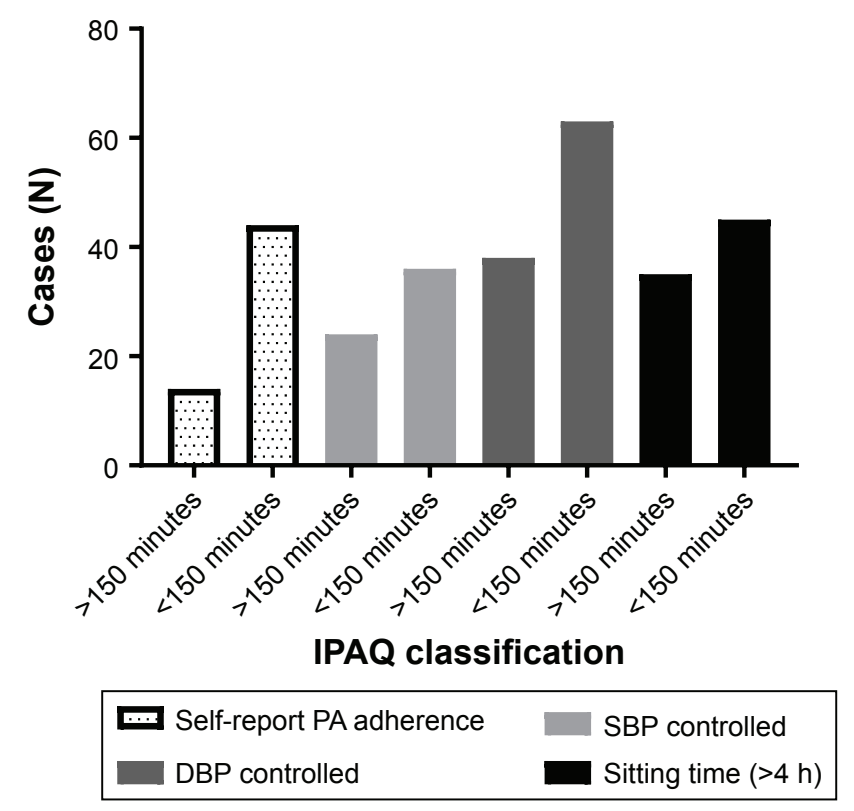

Figure I Number of cases for PA adherence, blood pressure control, and sitting time according to IPAQ classification.

Abbreviations: IPAQ, International Physical Activity Questionnaire; PA, physical activity.
Also, we did not find an association between inactivity (estimated by the sitting time) and BP control. The risk of inactivity for cardiovascular disease and uncontrolled BP is not clear. Some studies ${ }^{25,26}$ show a positive association between sedentary behavior (like use of computer and passive transportation) and sitting time with rates of hypertension. In a meta-analysis by Pandey et al, ${ }^{27}$ nonlinear association was found between sedentary time and the risk for CVD with increased risk only at very high levels ( $>10$ hours; HR 1.08; $95 \%$ CI, $100-1.14)$. In the same meta-analysis, ${ }^{27}$ a sensitivity analysis considering studies in which sedentary behavior was evaluated using accelerometer, no association was found between time spent on sedentary behavior and SBP or DBP. However, there was a positive association with sedentary behavior measured by screen time, TV time, computer, and sitting time. The different results may be attributed to different sources, like evaluation methods, memory bias, even samples characteristics, such as data from general population and studies with children included in meta-analysis. Anyway, the disputable association between prolonged inactivity and BP may be influenced by evaluation bias.

The main limitation of our study was the lack of objective assessment of PA such as accelerometers, which was not possible due to logistic issues. Although the better the precision of accelerometers, it is not widely used in clinical practice. Also, data collection based on one center reduces the external validity of our findings - we acknowledge that our results are partially explained by our patients' profile and peculiarities of our ambulatory.

In conclusion, the agreement between self-reported adherence for PA and IPAQ interview was low. The recommendation to engage in PA has low BP-lowering effectiveness in clinical setting, independent of the method of assessing adherence to the recommendations. Therefore, we believe that medical advice alone is not able to translate the efficacy of supervised PA demonstrated in clinical trials to clinical practice. 


\section{Acknowledgments}

The authors thank Vania N Hirakata, Biostatistics Unit, GPPG, Hospital de Clínicas de Porto Alegre. This work was supported by the Doctoral scholarship CAPES for GRR; Post-Doctoral scholarship CNPq for PABR; Fundação do Ministério da Educação (MEC), and Research Funding of Hospital de Clínicas de Porto Alegre (FIPE-HCPA), Brazil. The funding bodies had no role in the design of the study and collection, analysis, and interpretation of data or writing the manuscript.

\section{Author contributions}

GRR, PABR, and LBM contributed to conception and design of the research; GRR, GBM, AGS, and MPR helped in acquisition of data; GSN, VCJ, and SCF analyzed and interpreted the data and contributed to critical revision of the manuscript for intellectual content; GRR, PABR, LBM, and FDF statistically analyzed and wrote the manuscript. This study was equally supervised by LBM and PABR. All authors contributed to data analysis, drafting and revising the article, gave final approval of the version to be published, and agree to be accountable for all aspects of the work.

\section{Disclosure}

The authors report no conflicts of interest in this work.

\section{References}

1. World Health Organization. Global Health Observatory (GHO) data. Available from: http://www.who.int/gho/ncd/risk_factors/blood_ pressure_prevalence/en/. Accessed January 13, 2017.

2. Picon RV, Fuchs FD, Moreira LB, Riegel G, Fuchs SC. Trends in prevalence of hypertension in Brazil: a systematic review with meta-analysis. PLoS One. 2012;7(10):e48255.

3. Chobanian AV, Bakris GL, Black HR, et al. Seventh Report of the Joint National Committee on Prevention, Detection, Evaluation, and Treatment of High Blood Pressure. Hypertension. 2003;42(6):1206-1252.

4. Whelton PK, Carey RM, Aronow WS, et al. 2017 ACC/AHA/AAPA/ $\mathrm{ABC} / \mathrm{ACPM} / \mathrm{AGS} / \mathrm{APhA} / \mathrm{ASH} / \mathrm{ASPC} / \mathrm{NMA} / \mathrm{PCNA}$ guideline for the prevention, detection, evaluation, and management of high blood pressure in adults: executive summary: a report of the American College of Cardiology/American Heart Association Task Force on clinical practice guidelines. Hypertension. 2018;71(6):1269-1324.

5. Group LW. US Department of Health and Human Services: Lifestyle interventions to reduce cardiovascular risk; 2013. Available from: https:// www.nhlbi.nih.gov/sites/default/files/media/docs/lifestyle.pdf. Accessed March 03, 2018

6. Pescatello LS, Franklin BA, Fagard R, et al. American College of Sports Medicine position stand. Exercise and hypertension. Med Sci Sports Exerc. 2004;36(3):533-553.

7. Vanhees L, Geladas N, Hansen D, et al. Importance of characteristics and modalities of physical activity and exercise in the management of cardiovascular health in individuals with cardiovascular risk factors: recommendations from the EACPR. Part II. Eur J Prev Cardiol. 2012;19(5):1005-1033.

8. Brook RD, Appel LJ, Rubenfire M, et al. Beyond medications and diet: alternative approaches to lowering blood pressure: a scientific statement from the American Heart Association. Hypertension. 2013;61(6):1360-1383.
9. Whelton SP, Chin A, Xin X, He J. Effect of aerobic exercise on blood pressure: a meta-analysis of randomized, controlled trials. Ann Intern Med. 2002;136(7):493-503.

10. Bravata DM, Smith-Spangler C, Sundaram V, et al. Using pedometers to increase physical activity and improve health: a systematic review. JAMA. 2007;298(19):2296-2304.

11. Cornelissen VA, Fagard RH, Coeckelberghs E, Vanhees L. Impact of resistance training on blood pressure and other cardiovascular risk factors: a meta-analysis of randomized, controlled trials. Hypertension. 2011;58(5):950-958.

12. Owen A, Wiles J, Swaine I. Effect of isometric exercise on resting blood pressure: a meta analysis. J Hum Hypertens. 2010;24(12):796-800.

13. Moreira WD, Fuchs FD, Ribeiro JP, Appel LJ. The effects of two aerobic training intensities on ambulatory blood pressure in hypertensive patients: results of a randomized trial. J Clin Epidemiol. 1999;52(7): 637-642.

14. Andrews RC, Cooper AR, Montgomery AA, et al. Diet or diet plus physical activity versus usual care in patients with newly diagnosed type 2 diabetes: the Early ACTID randomised controlled trial. Lancet. 2011;378(9786):129-139.

15. Williamson W, Foster C, Reid H, et al. Will exercise advice be sufficient for treatment of young adults with prehypertension and hypertension? A systematic review and meta-analysis. Hypertension. 2016;68(1): 78-87.

16. Fuchs FD, Gus M, Moreira WD, et al. Blood pressure effects of antihypertensive drugs and changes in lifestyle in a Brazilian hypertensive cohort. J Hypertens. 1997;15(7):783-792.

17. Riegel G, Moreira LB, Fuchs SC, et al. Long-term effectiveness of non-drug recommendations to treat hypertension in a clinical setting. Am J Hypertens. 2012;25(11):1202-1208.

18. Craig CL, Marshall AL, Sjöström M, et al. International Physical Activity Questionnaire: 12-country reliability and validity. Med Sci Sports Exerc. 2003;35(8):1381-1395.

19. Dempsey PC, Owen N, Biddle SJ, Dunstan DW. Managing sedentary behavior to reduce the risk of diabetes and cardiovascular disease. Curr Diab Rep. 2014;14(9):522.

20. Van Holle V, De Bourdeaudhuij I, Deforche B, Van Cauwenberg J, Van Dyck D. Assessment of physical activity in older Belgian adults: validity and reliability of an adapted interview version of the long International Physical Activity Questionnaire (IPAQ-L). BMC Public Health. 2015;15:433.

21. Strath SJ, Kaminsky LA, Ainsworth BE, et al. Guide to the assessment of physical activity: clinical and research applications: a scientific statement from the American Heart Association. Circulation. 2013; 128(20):2259-2279.

22. Corso LM, Macdonald HV, Johnson BT, et al. Is concurrent training efficacious antihypertensive therapy? A meta-analysis. Med Sci Sports Exerc. 2016;48(12):2398-2406.

23. Huebschmann AG, Reis EN, Emsermann C, et al. Women with type 2 diabetes perceive harder effort during exercise than nondiabetic women. Appl Physiol Nutr Metab. 2009;34(5):851-857.

24. Riegel B, Dickson VV, Cameron J, et al. Symptom recognition in elders with heart failure. J Nurs Scholarsh. 2010;42(1):92-100.

25. Lee PH, Wong FK. The association between time spent in sedentary behaviors and blood pressure: a systematic review and meta-analysis. Sports Med. 2015;45(6):867-880.

26. Ekelund U, Steene-Johannessen J, Brown WJ, et al. Does physical activity attenuate, or even eliminate, the detrimental association of sitting time with mortality? A harmonised meta-analysis of data from more than 1 million men and women. Lancet. 2016;388(10051):1302-1310.

27. Pandey A, Salahuddin U, Garg S, et al. Continuous dose-response association between sedentary time and risk for cardiovascular disease: a meta-analysis. JAMA Cardiol. 2016;1(5):575-583. 
Patient Preference and Adherence

Dovepress

\section{Publish your work in this journal}

Patient Preference and Adherence is an international, peer-reviewed, open access journal that focuses on the growing importance of patient preference and adherence throughout the therapeutic continuum. Patient satisfaction, acceptability, quality of life, compliance, persistence and their role in developing new therapeutic modalities and compounds to optimize

clinical outcomes for existing disease states are major areas of interest for the journal. This journal has been accepted for indexing on PubMed Central. The manuscript management system is completely online and includes a very quick and fair peer-review system, which is all easy to use. Visit http://www. dovepress.com/testimonials.php to read real quotes from published authors.

Submit your manuscript here: http://www.dovepress.com/patient-preference-and-adherence-journal 